\title{
Distinct roles of Akt1 in regulating proliferation, migration and invasion in HepG2 and HCT 116 cells
}

\author{
LIANG CHEN $^{1}$, QIAO-HUI KANG ${ }^{1}$, YING CHEN $^{1}$, YA-HONG ZHANG ${ }^{2}$, \\ QIAN LI ${ }^{2}$, SONG-QIANG XIE ${ }^{1}$ and CHAO-JIE WANG ${ }^{2}$ \\ ${ }^{1}$ Institute of Chemical Biology, Henan University, Kaifeng, Henan 475004; ${ }^{2}$ The Key Laboratory \\ of Natural Medicine and Immuno-Engineering, Henan University, Kaifeng, Henan 475004, P.R. China
}

Received October 22, 2013; Accepted November 18, 2013

DOI: $10.3892 / o r .2013 .2879$

\begin{abstract}
Elucidating the effects of genes involved in tumors may improve therapeutic strategies for human cancer. Recently, several studies discovered that Aktl plays a dual role in mediating cell proliferation, migration and invasion, depending on the cell type. However, the pathophysiological role of Akt1 in hepatocellular carcinoma (HCC) and colorectal carcinoma cells remains poorly understood. In the present study, we transfected the Akt1-expressing plasmids into the tumor cells that expressed only low levels of Akt1. The migration and invasion abilities were analyzed in 24-well Boyden chambers. The expression of proteins was detected using western blot analysis. Our results demonstrated that overexpression of Akt1 significantly enhanced the proliferation rates and promoted the colony formation in both HepG2 and HCT 116 cells. When treated with wortmannin, the ability to form colonies was significantly attenuated in both cell lines. Of note, enforced expression of Akt1 induced HepG2 cell migration and invasion; by contrast, upregulation of Akt1 expression suppressed the migration and invasion of HCT 116 cells. Subsequent mechanistic investigations revealed that upregulation of Akt1 markedly induced the expression of Bcl- 2 and NF- $\kappa \mathrm{B}$ in both types of tumor cells. Notably, we observed a similar increase of MMP2, MMP9, HIF1 $\alpha$ and VEGF in HCC cells, whereas Akt1 significantly suppressed the expression of these molecules in colorectal carcinoma cells. These data suggest a dual role for Aktl in tumor cell migration and invasion and highlight the cell type-specific actions of Aktl kinases in the regulation of cell motility.
\end{abstract}

Correspondence to: Professor Chao-Jie Wang, The Key Laboratory of Natural Medicine and Immuno-Engineering, Henan University, Kaifeng, Henan 475004, P.R. China

E-mail: wcjsxq@henu.edu.cn

Professor Song-Qiang Xie, Institute of Chemical Biology, Henan University, Kaifeng, Henan 475004, P.R. China

E-mail: xiesq@henu.edu.cn

Key words: Akt1, wortmannin, proliferation, migration, invasion

\section{Introduction}

The phosphoinositide 3-kinase (PI3K) signaling pathway regulates a multitude of cellular processes including cell survival, proliferation, migration and invasion. Deregulation of the PI3K signaling pathway is often detected in various types of human cancer. The serine/threonine kinase Akt, also known as protein kinase B (PKB), which was initially identified as a proto-oncogene Akt8, from a spontaneous thymoma of an AKR mouse (1), has a strong oncogenic function and is the primary downstream mediator of PI3K pathway function (2). In mammals, there are three different isoforms of Akt, termed Akt1, Akt2 and Akt3, which share a high degree of homology, while the three Akt isoforms have some different functions (3).

Amplification of Akt1 was first discovered in a primary human gastric adenocarcinoma (1). Currently, increasing evidence indicates that Akt1 has been implicated in the control of various biological processes, including cell proliferation, survival and tumor formation. Menges et al (4) demonstrated that Akt1 is positively correlated with tumor cell proliferation and survival. Downregulation of Akt1 expression inhibits K562 cell proliferation (5). Furthermore, by using bitransgenic MMTV-c-ErbB2, MMTV-myr-Akt1 mouse models, constitutively active Akt1 markedly accelerated MMTV-c-ErbB2 mammary tumorigenesis (6). However, the role of Akt1 in cell proliferation remains controversial. For instance, a recent article indicated that constitutive Akt1 signals attenuate B-cell receptor signaling and proliferation (7). Therefore, it is essential to investigate whether ectopic expression of Akt1 promotes cell proliferation or not in other cell types, including hepatocellular carcinoma (HCC) and colorectal cancer cells.

Cell migration and invasion are two of the most important steps involved in cancer metastasis, which accounts for $>90 \%$ of all cancer-related deaths $(8,9)$. Growing evidence indicates that activation of Akt1 is associated with cancer cell migration, invasion and metastasis. For example, selective activation of Akt1 not only positively regulates IGF-1-induced SKOV-3 cell migration and invasion, but also markedly promotes tumor metastasis (10). Previous studies showed that Akt isoform Akt1 limits breast cancer cell motility and invasion $(11,12)$ and several other independent studies have validated and extended these observations $(13,14)$. Moreover, 
these data have been validated in transgenic mouse models, in which AKT1 was shown to have an inhibitory effect on breast cancer invasiveness $(15,16)$. Although overwhelming evidence has demonstrated that the PI3K/Akt signaling cascade plays a crucial role in the regulation of the malignant behaviors, including migration and invasion in $\mathrm{HCC}$ and colorectal carcinomas, the role of Akt1 and its precise molecular mechanisms in these cancer cells remain largely unknown.

In the present study, we demonstrated that upregulation of Akt1 significantly increased cell proliferation and enhanced the ability to form colonies in both HepG 2 and HCT 116 cells. Furthermore, we also discovered that enforced expression of Akt1 significantly enhanced the ability of migration and invasion in HepG2 cells, while it reduced HCT 116 cell migration and invasion. These data demonstrate a dual role for Akt1 in tumor cell migration and invasion and highlight the cell type-specific actions of Aktl kinases in the regulation of cell motility.

\section{Materials and methods}

Materials. 3-(4, 5-dimethylthiazol)-2,5-diphenyltetrazolium bromide (MTT), G418 and wortmannin were purchased from Sigma (St. Louis, MO, USA). RPMI-1640 and fetal calf serum (FCS) were purchased from Gibco (Grand Island, NY, USA). The sources of primary antibodies used for western blot analysis, Akt1, Bcl-2, NF-кB, MMP2, MMP9, HIF1 $\alpha$, VEGF, as well as horseradish peroxidase-conjugated anti-mouse and anti-rabbit antibodies were all purchased from Santa Cruz Biotechnology (Santa Cruz, CA, USA). All other chemicals used in the experiments were commercial products of reagent grade.

Cell culture and treatment. Cell lines from human HCC (HepG2) and colorectal carcinoma (HCT 116) were purchased from the Cell Bank of the Chinese Academy of Science (Shanghai, China). These cells were maintained in RPMI-1640 medium supplemented with $10 \%$ FCS, $100 \mathrm{U} / \mathrm{ml}$ penicillin, and $100 \mu \mathrm{g} / \mathrm{ml}$ streptomycin at $37^{\circ} \mathrm{C}$ in a humidified incubator containing $5 \% \mathrm{CO}_{2}$.

Plasmid construction and transfection. The full-length coding region (1443 bp) of Aktl was amplified from human genomic DNA by reverse transcription-polymerase chain reaction (RT-PCR) using the following primers: Akt1-sense 5'-CCG CTC GAG ACC ATG AGC GAC GTG GCT ATT GTG AAG-3', and antisense 5'-CGG GAT CCG CAG TCC ACC GCC GCC TCAG-3', and the PCR products were then digested with $\mathrm{XhoI} / \mathrm{Bam} \mathrm{HI}$ and were inserted into the pIRES2EGFP vector (Clontech Laboratories, Inc., Palo Alto, CA, USA) carrying neomycin resistance gene. The recombinant construct was verified by direct DNA sequencing.

For transfection, HepG2 and HCT 116 cells were seeded in 24-well plates at $5 \times 10^{4} /$ well and incubated at $37^{\circ} \mathrm{C}$ in a humidified incubator containing $5 \% \mathrm{CO}_{2}$. The next day, when these cells were $\sim 80 \%$ confluent, cells were transfected with TurboFect ${ }^{\mathrm{TM}}$ in vitro transfection reagent according to the manufacturer's instructions. Briefly, recombinant plasmid $(1 \mu \mathrm{g})$ was mixed with TurboFect ${ }^{\mathrm{TM}}$ reagent and pre-incubated for $20 \mathrm{~min}$ at room temperature in $100 \mu \mathrm{l}$ of serum-free RPMI-1640. HepG2 and HCT 116 cells transfected with pIRES2-EGFP-Akt1 and pIRES2-EGFP vector were termed as HepG2-Akt1 and HepG2-Vec, HCT 116-Akt1 and HCT 116-Vec, respectively. These transfected cells were selected in the presence of G418 $(100 \mu \mathrm{g} / \mathrm{ml})$ for 2 weeks, and then the stable plasmid-transfected clones were generated by using limiting dilution analyses in 96-well plates.

Cell growth curve analysis. The MTT assay was used to detect the proliferation rate of cells transfected with empty vector or Akt1 plasmid. The process was performed as previously described with some modifications (17). Briefly, 2,000 cells/well were plated in 96-well plates and incubated for 1, 2, 3, 4, 5, 6 and 7 days, respectively. Then, $50 \mu 1$ MTT reagents $(1 \mathrm{mg} / \mathrm{ml})$ was added at indicated time-points and cells were incubated for $4 \mathrm{~h}$ at $37^{\circ} \mathrm{C}$. Supernatants were removed from the wells and $100 \mu \mathrm{l}$ DMSO was pipetted to solubilize the crystal product for $10 \mathrm{~min}$ at room temperature. The absorbance (OD) of each well was measured with a microplate reader (Bio-Rad Laboratories) at a wavelength of $570 \mathrm{~nm}$.

RNA isolation and RT-PCR. Total RNA was extracted using TRIzol reagent (Invitrogen). Briefly, $2 \mu \mathrm{g}$ of total RNA was subjected to DNase I digestion (1 U/ $\mu \mathrm{l}$; Fermentas, Hanover, $\mathrm{MD}, \mathrm{USA})$ at $37^{\circ} \mathrm{C}$ for $30 \mathrm{~min}$ and then to heat inactivation of DNase $\mathrm{I}$ at $70^{\circ} \mathrm{C}$ for $15 \mathrm{~min}$, followed by reverse-transcription using Moloney murine leukemia virus reverse transcriptase (Promega). The PCR primers and regimen were: 5'-ATG AGC GAC GTG GCT ATT GTG AAG-3' and 5'-GAG GCC GTC AGC CAC AGT CTG GATG-3' for Akt1 (330 bp); 5'-CGG AGT CAA CGG ATT GGT CGT AT-3', and 5'-AGC CTT CTC CAT GGT GG TGA AGAC-3' for GAPDH (307 bp). All PCR reactions were performed using standard PCR conditions: $95^{\circ} \mathrm{C}$ for $5 \mathrm{~min}, 95^{\circ} \mathrm{C}$ for $45 \mathrm{sec}$, annealing at different temperatures for each gene respectively for $45 \mathrm{sec}$, extension at $72^{\circ} \mathrm{C}$ for $1 \mathrm{~min}$ for 30 cycles, and a final extension at $72^{\circ} \mathrm{C}$ for $10 \mathrm{~min}$. PCR products were separated on $1.0 \%$ agarose gel. The gel was then digitally photographed and scanned with UVI Gel Analyzing System (UVI Tech, Cambridge, UK).

Colony formation assay. Cells were plated in a fresh 24-well plate at a density of 200 cells/well and maintained in RPMI-1640 containing $10 \%$ FCS. The medium was changed every 3 days for 14 days until visible colonies formed. Colonies were fixed and stained with $0.1 \%$ crystal violet in $20 \%$ methanol for $15 \mathrm{~min}$. Individually stained colonies ( $>50$ cells) were counted in each well. The colony formation assay was performed as previously reported with some modifications (18).

Wound scratch assay. The wound scratch assay was performed as previously described (19). Briefly, cells were grown to confluence overnight prior to serum starvation for $24 \mathrm{~h}$. The confluent cell monolayer was then scratched with a pipette tip $(20 \mu \mathrm{l})$ and washed thrice with PBS to remove floating cells. After the line scratch, the width of the wound was measured and recorded as $t=0$. The cells were then allowed to migrate back into the wounded area and the closing of the wound was measured at $24 \mathrm{~h}$. The migration distance (in $\mu \mathrm{m}$ ) was determined as the reduction of the width of the open area. 
Boyden chamber assay. The migration and invasion assays were performed in 24-well Boyden chambers with $8 \mu \mathrm{m}$ pore size polycarbonate membranes (Corning, Corning, NY, USA), as previously described (20). For the invasion assay, the membrane was coated with $15 \mu \mathrm{g}$ Matrigel (R\&D Systems) to form a matrix barrier. Serum-starved cells ( $1 \times 10^{5}$ cells) were seeded into the upper compartment of the chamber in serum-free medium, supplemented with $100 \mathrm{nM}$ wortmannin or not, while the lower compartment was filled with $600 \mu \mathrm{l}$ of DMEM containing $10 \% \mathrm{FBS}$. Following incubation at $37^{\circ} \mathrm{C}$ for $24 \mathrm{~h}$, tumor cells remaining on the upper surface of the membrane were removed with cotton swabs. The cells on the lower surface of the membrane were fixed, stained with crystal violet and then counted under a light microscope. For the migration assay, only one third of cells was applied to the Transwell chamber without Matrigel.

Western blot analysis. After treatment with $100 \mathrm{nM}$ wortmannin or not for $24 \mathrm{~h}$, cells were harvested and washed with PBS. Cell lysates were prepared in the protein extraction buffer containing $150 \mathrm{mM} \mathrm{NaCl}, 10 \mathrm{mM}$ Tris (pH 7.2), $5 \mathrm{mM}$ EDTA, $0.1 \%$ Triton X-100, 5\% glycerol and 2\% SDS. Western blot analysis was performed as previously described (21). The total protein concentration was determined using the protein assay kit (Beyotime, China). Cell lysates in 5X SDS-sample buffer were boiled for $5 \mathrm{~min}$ and then equal amounts of total proteins were separated using 10 or $12 \%$ SDS-PAGE and transferred to polyvinylidene difluoride (PVDF) membranes (Immobilion Millipore). Membranes were then blocked in PBST containing 5\% dried skimmed milk for $1 \mathrm{~h}$ at room temperature. The blots were incubated with corresponding primary antibodies at $4^{\circ} \mathrm{C}$ overnight. After washing three times with PBST, the membranes were incubated with corresponding horseradish peroxidase-conjugated goat anti-mouse or anti-rabbit secondary antibody, and then washed three times with PBST. Proteins were detected using the ECL plus reagents (Beyotime, China).

Statistical analysis. Data are expressed as the means \pm SD from at least three independent experiments. Unless otherwise noted, the differences between groups were assessed by ANOVA. All the tests performed in the present study were two-sided using SPSS 13.0 software (SPSS Inc., Chicago, IL, USA). Differences were considered statistically significant at values of $\mathrm{P}<0.05$.

\section{Results}

Akt1 is stably overexpressed in HepG2 and HCT 116 cells. After stably transfected cells were individually selected, the Akt1 and GAPDH mRNA levels were measured using RT-PCR. Compared with the control group, the pIRES2EGFP-Akt1 transcripts were strongly upregulated (Fig. 1A and B). Consistent with our RT-PCR data, western blot analysis revealed that Akt1 protein levels were also clearly increased in pIRES2-EGFP-Akt1-transfected cells (Fig. 1C and D). The pIRES2-EGFP empty vector did not substantially affect the endogenous Akt1 expression in both mRNA and protein levels. Collectively, these findings indicate that Akt1 is stably overexpressed in HepG2 and HCT 116 cells.
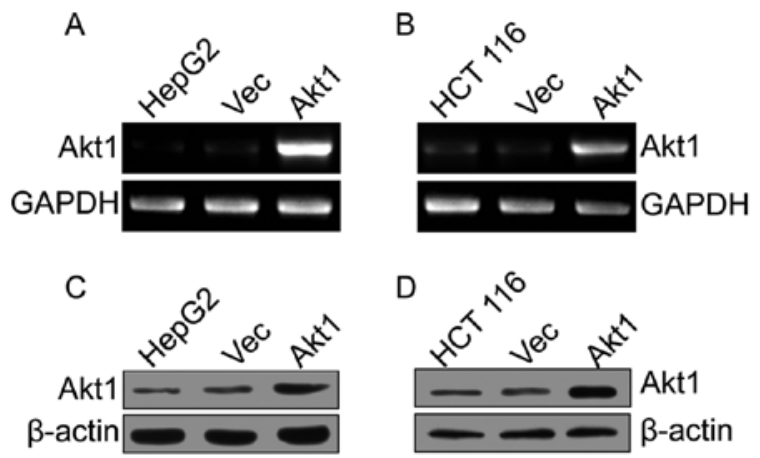

Figure 1. Overexpression of Akt1 detected in transfections. Construction of the recombinant eukaryotic expression vector pIRES2-EGFP containing the Aktl gene was used in the studies. Transfection of the recombinant plasmid pIRES2-EGFP-Akt1 or pIRES2-EGFP empty vector into HepG2 and HCT 116 cells, respectively. (A and B) Semi-quantitative RT-PCR analysis was used to identify the Akt1 mRNA level in tumor cells. GAPDH was used as a loading control. (C and D) Western blot analysis was used to determine the Akt1 protein level in various tumor cells; $\beta$-actin was used as an internal control.

Aktl upregulation promotes cell proliferation and colony formation in HepG2 and HCT 116 cells. We then determined the functional consequences of Akt1. As shown in Fig. 2A and $\mathrm{B}$, compared with the controls, the proliferation rates of HepG2-Akt1 and HCT 116-Akt1-transfected cells were increased significantly $(\mathrm{P}<0.01)$; then, the capacity of colony formation was evaluated on these cell lines. Our results showed that the number of colonies of HepG2-Akt1 and HCT 116-Akt1 cells were $66.7 \pm 9.6$ and $71.7 \pm 5.9$, respectively; however, the number of colonies was only $29.3 \pm 4.7$ and 29.0 \pm 6.0 for HepG2-Vec and HCT 116-Vec cells, respectively. There was a statistically significant increase in the number of colonies of HepG2-Akt1 and HCT 116-Akt1 cells compared to that of the individual control groups (Fig. $2 \mathrm{C}$ and $\mathrm{D} ; \mathrm{P}<0.01$, $\mathrm{P}<0.001$, while wortmannin significantly attenuated the colonies of the tumor cells with upregulation of Akt1. These results indicate that Akt1 promotes tumor cell proliferation and colony formation.

Upregulation of Akt1 expression promotes HepG2 cell migration and invasion. Next, we examined the effect of Akt1 on HepG2 cell migration and invasion. As shown in Fig. 3A, a significant difference between wound distance of HepG2-Akt1 cells compared to HepG2-Vec cells was observed. Forced expression of Akt1 markedly stimulated wound closure compared with the empty vector-transfected cells, while this effect was significantly decreased after treatment with wortmannin (Fig. 3A). Boyden chamber Transwell assay also showed that the migration of HepG 2 cells was increased by $>2$-fold due to Aktl overexpression, but was significantly decreased when treated with wortmannin (Fig. 3C).

To determine whether Akt1 also regulates HepG2 cell invasion, the Transwell assay was repeated. As shown in Fig. 4A, upregulation of Aktl expression markedly induced cell invasion compared with the control group, whereas cell invasion was strongly suppressed by wortmannin. Taken together, these results demonstrate that Aktl is correlated with cell migration and invasion potential of liver cancer HepG2 cells. 
A

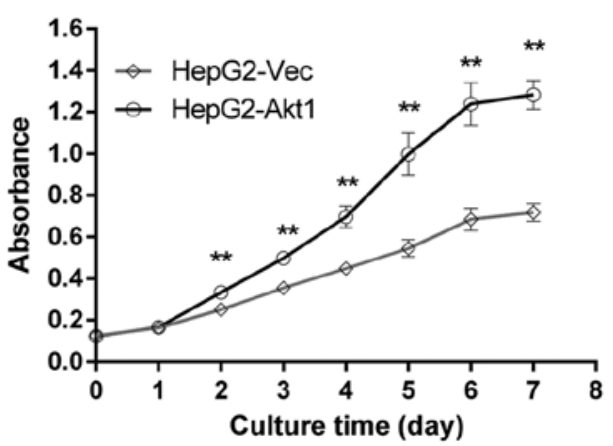

B

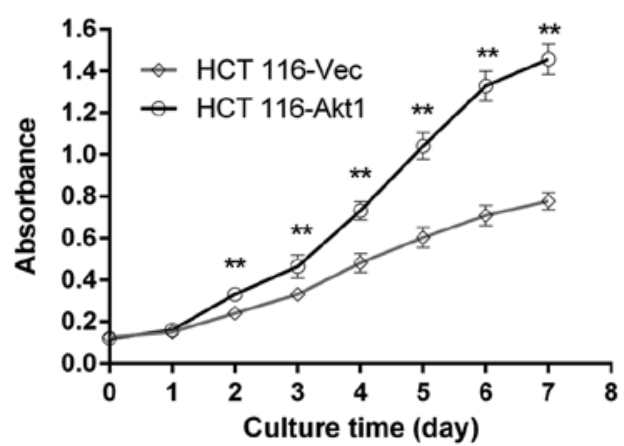

C
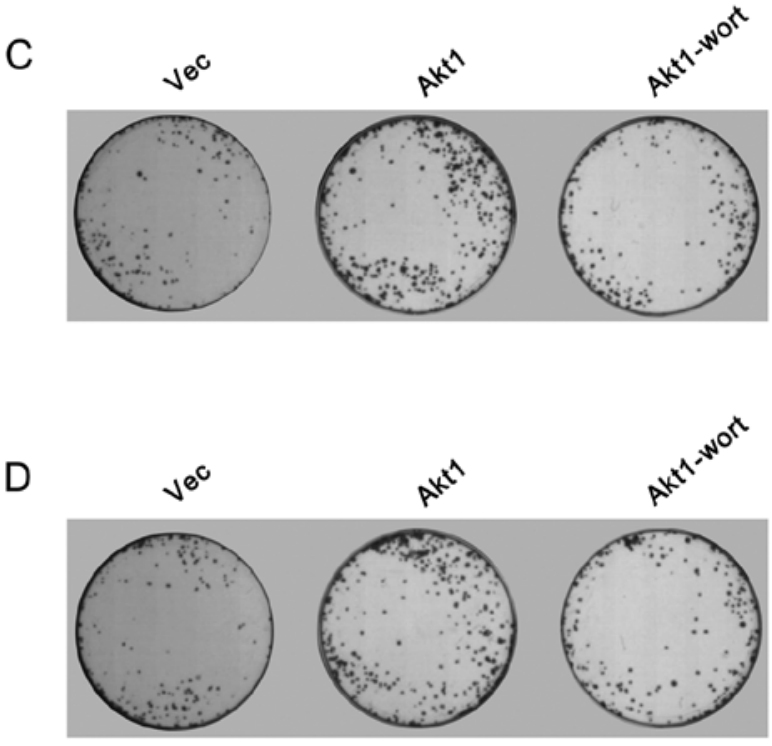

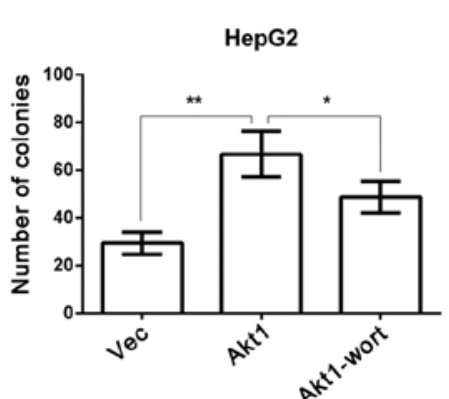

HCT 116

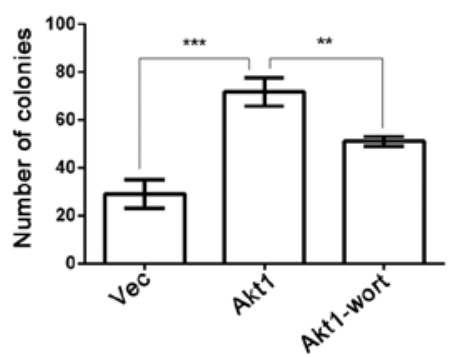

Figure 2. Upregulation of Akt1 promotes tumor cell proliferation and colony formation. (A and B) MTT assay showed that Akt1 overexpression promoted the cell proliferation rates in both HepG2 and HCT 116 cell lines. (C and D) Upregulation of Akt1 expression increased the number of colony formation in HepG2 and HCT 116 cells. Cells were incubated in the absence or presence of $100 \mathrm{nM}$ wortmannin (wort) for $24 \mathrm{~h}$, which was then changed with fresh medium. Colony numbers ( $>50$ cells/colony) were counted at day 15 . These results were reproducible in three independent experiments. ${ }^{*} \mathrm{P}<0.05 ;{ }^{* * *} \mathrm{P}<0.01,{ }^{* * * *} \mathrm{P}<0.001$.

Overexpression of Aktl suppresses the migration and invasion of HCT 116 cells. We determined the role of Akt1 in HCT 116 cell migration. Upregulation of Akt1 expression markedly suppressed wound closure compared with HCT 116-Vec cells, while wortmannin treatment significantly reversed the effects of Aktl on HCT 116 cells (Fig. 3B). The Transwell assay also indicated that ectopic expression of Akt1 noticeably decreased HCT 116-Akt1 cell migration by $\sim 50 \%$ in comparison with the control group (Fig. 3D). The addition of wortmannin significantly attenuated the inhibitory effect of Akt1 on HCT 116 cell migration (Fig. 3D). We then examined whether Aktl also regulates HCT 116 cell invasion. Akt1 overexpression significantly inhibited invasion compared to control cells transfected with empty vector (Fig. 4B), while wortmannin partially antagonized the inhibitory effect of Akt1 on migration and invasion of HCT 116 cells (Fig. 4B). These findings suggest that Akt1 suppresses the cell migration and invasion of HCT 116 cells.

Cellular signaling pathways involved in Aktl action. To identify the cellular signaling pathways involved in Akt1 action, we first examined the pro-survival and proliferative molecules downstream of Akt1, including Bcl-2 and nuclear factor- $\kappa \mathrm{B}$ $(\mathrm{NF}-\kappa \mathrm{B})$. Overexpression of Akt1 significantly induced the expression of Bcl-2 and NF- $\kappa \mathrm{B}$ in both HepG2 (Fig. 5A) and HCT 116 cells (Fig. 5B). However, wortmannin partly reversed Akt1-mediated effects.

Overexpression of MMPs is associated with tumor invasion and metastasis and, in particular, MMP2 and MMP9, which are well known to play a pivotal role in tumor invasion and metastasis development in various types of cancer including hepatocellular and colorectal carcinomas $(22,23)$. Our results also showed that Akt1 overexpression significantly increased the expression of MMP2 and MMP9 in HepG2 cells, while there was an opposing effect of Akt1 in HCT 116 cells. Of note, wortmannin significantly attenuated these effects of Akt1 in both types of tumor cells (Fig. 5). Our results also demonstrated that upregulation of Akt1 also enhanced the expression of HIF-1 $\alpha$ and VEGF in HepG2 cells, while it attenuated the levels of these two types of proteins in HCT 116 cells. 

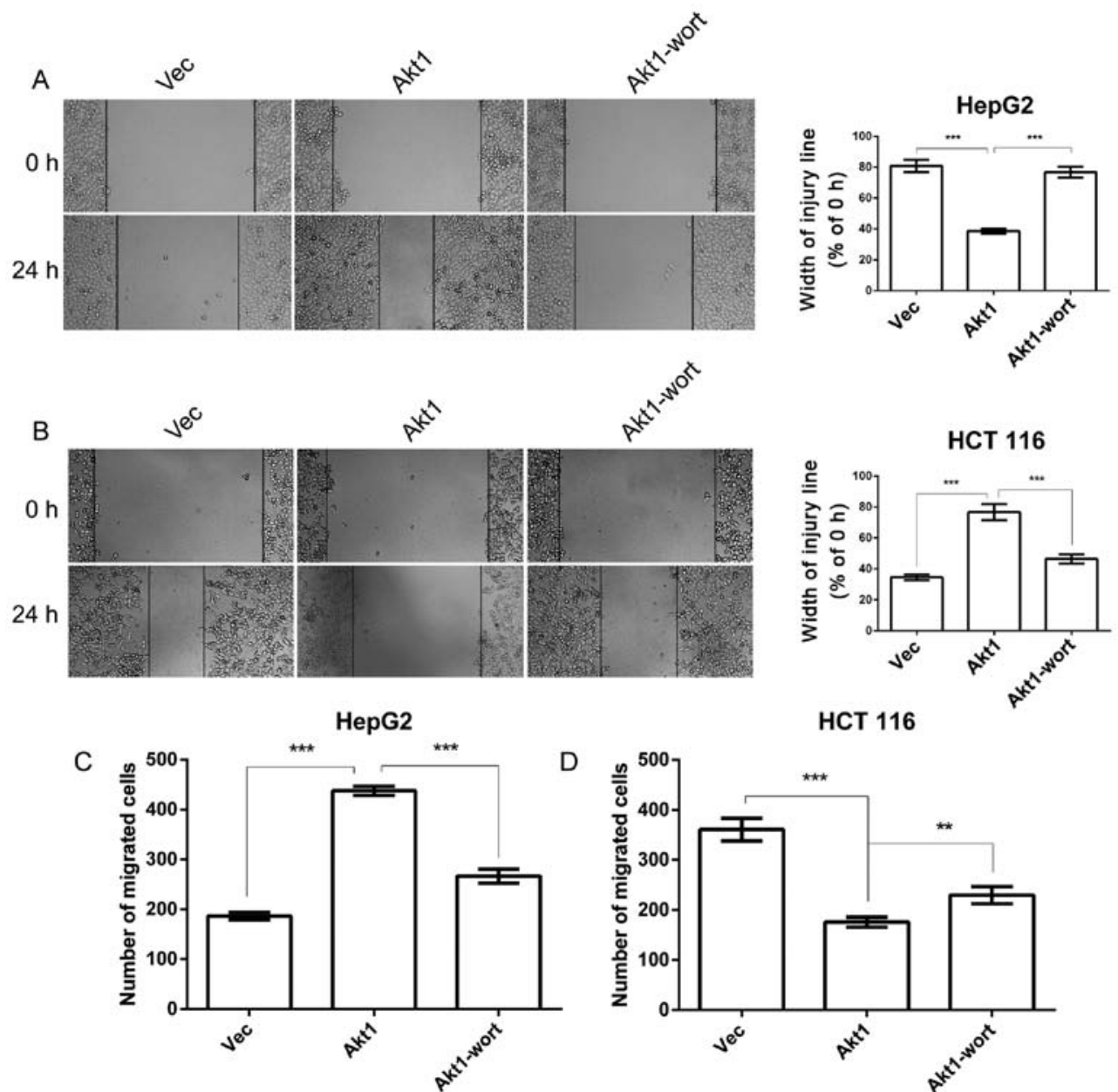

Figure 3. The effects of Akt1 on cancer cell migration. (A and C) Effect of Akt1 on HepG2 cell migration was detected by wound scratch assay and Transwell chamber without Matrigel. Representative results of migration of Vec-transfected and Akt1-transfected HepG2 cells that were treated with $100 \mathrm{nM}$ wortmannin (wort), or that were left untreated for $24 \mathrm{~h}$, and then photographed. (B and D) Effect of Akt1 on HCT 116 cell migration was performed using wound scratch assay and Transwell chamber without Matrigel. Representative results of migration of the HCT 116 cell lines that were transfected with empty vector or Akt1. These cells were incubated with or without $100 \mathrm{nM}$ wort for $24 \mathrm{~h} .{ }^{* *} \mathrm{P}<0.01{ }^{* * * *} \mathrm{P}<0.001$.

A

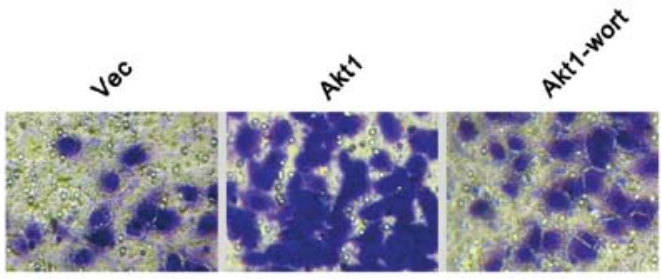

B

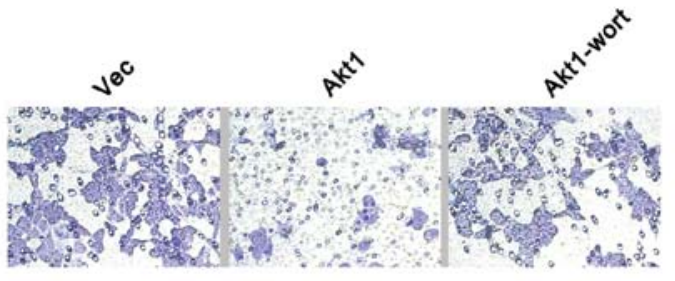

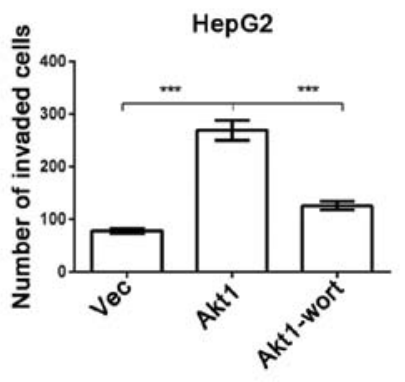

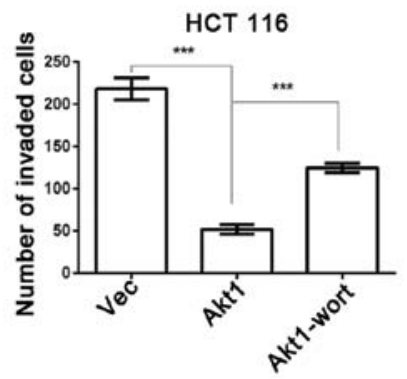

Figure 4. The effects of Akt1 on tumor cell invasion. (A) Akt1 promoted the invasion of HepG2 cells. (B) Akt1 upregulation attenuated the invasion of HCT 116 cells. Representative results of invasion of Vec-transfected and Akt1-transfected HepG2 and HCT 116 cells that were treated with $100 \mathrm{nM}$ wortmannin (wort) or that were left untreated. These cells were then added to Transwell chamber coated with Matrigel and incubated for $24 \mathrm{~h}$. Viability of cell invasion was expressed by the number of invading cells. ${ }^{* * *} \mathrm{P}<0.001$. 


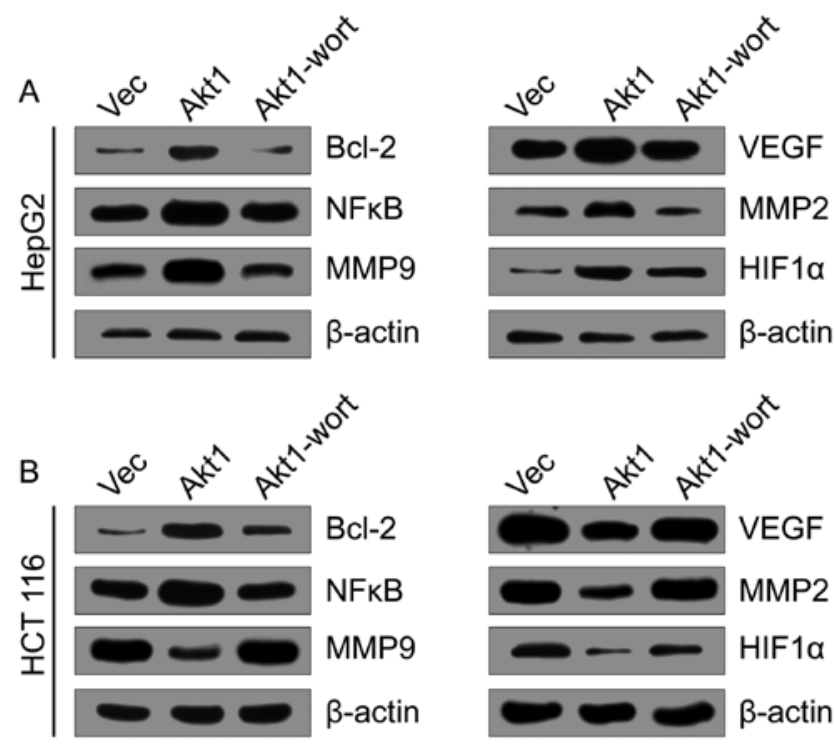

Figure 5. The effects of Akt1 on cell signaling pathways. Western blot results for the expression of Bcl-2, NF- $\mathrm{kB}$, MMP2 and MMP9, HIF1 $\alpha$ and VEGF. (A and B) Akt1 regulates the expression of various proteins. HepG2 and HCT 116 cells transfected with empty vector and Akt1 were cultured in the presence or absence of $100 \mathrm{nM}$ wortmannin (wort) for $24 \mathrm{~h}$, prior to immunoblotting analysis for these protein expressions. All results were repeated in three independent experiments and the representative immunoblots are shown; $\beta$-actin was used as an internal control.

\section{Discussion}

Activation of the PI3K/Akt signaling pathway has been detected in several types of cancer, and PI3K or its downstream components, including AKTs, are considered attractive targets for cancer therapy. However, several studies have highlighted that the biological outcomes obtained upon AKT inhibition are very complex $(13,24,25)$, including the potential cell-type specific effects of AKT isoform Akt1 on cell migration and invasion (10). Therefore, it is necessary to investigate the potential role of Aktl in various types of human cancer before its inhibitors are used as cancer therapies.

In the present study, our findings showed that upregulation of Akt1 resulted in increased cell proliferation in both HepG2 and HCT 116 cells. Inhibition of PI3K by wortmannin efficiently reduced the colonies in tumor cells with Akt1 transfection. Consistent with our findings, ablation of AKT1 decreased the proliferation of the androgen-independent cell line PC-3 (26). Moreover, as these are in vitro assays, validation of these results in vivo is required and only few reports have already addressed these issues. Using mice bitransgenic of mammary tumorigenesis with constitutively active Akt1 and ErbB-2, Young et al (6) reported that overexpression of activated Akt1 resulted in increased tumor frequency. In contrast, loss of Akt1 reduced tumor proliferative function in a murine model of thyroid cancer (27). These results suggested that Akt1 expression in tumor cells is required for cell proliferation, although how ectopic expression of Akt1 promotes tumor cell proliferation remains unclear.

We investigated the potential cellular signaling pathway involved in Akt1-induced cell proliferation. Bcl-2 is a wellknown pro-survival gene downstream of Akt1. It is often overexpressed in various human tumors. In the present study, our results indicated that $\mathrm{Bcl}-2$ is overexpressed in both HepG2-Akt1 and HCT 116-Akt1 cells compared with their respective vector-transfected cells. Upon wortmannin treatment, the expression of $\mathrm{Bcl}-2$ was significantly downregulated, suggesting that Bcl-2 may play a key role in Akt1-induced proliferation. In concordance with our observations, a recent study indicated that the $\mathrm{Akt1} / \mathrm{Bcl}-2$ signaling pathway plays a critical role in angiopoietin-2-stimulated cell survival and proliferation both in vitro and in vivo (28). Moreover, silencing of Aktl was associated with the suppression of Bcl-2 which, in turn, resulted in the inhibition of cell proliferation, stimulation of apoptosis in vitro and inhibition of xenograft growth in nude mice (29).

Nuclear factor- $\mathrm{\kappa B}$ (NF- $\mathrm{\kappa B}$ ) is also a ubiquitous transcription factor. It is often thought to contribute to malignant transformation by aberrant activation of cellular functions that are commonly associated with tumor promotion, including stimulating cell growth and proliferation (30). Furthermore, Han et al (31) supported the notion that constitutive activation of the PI3K/Akt1/NF- $\mathrm{KB}$ signaling pathway is important for cell survival and proliferation in $\mathrm{iMyc}{ }^{\mathrm{E} \mu} \mathrm{B}$-cell lymphomas. In the present study, enforced expression of Akt1 increased the expression of NF- $\mathrm{kB}$ in both HepG2 and HCT 116 cells. Upon wortmannin treatment, the level of NF- $\mathrm{kB}$ was markedly downregulated comparable to that of $\mathrm{HepG} 2-\mathrm{Vec}$ and HCT 116-Vec cells, respectively. Considering that, the expression of Bcl-2 and NF- $\kappa \mathrm{B}$ is in concordance with Akt1-mediated cell proliferation in both HepG2 and HCT 116 cells. Hence, we proposed that Bcl-2 and NF- $\mathrm{kB}$ are two important molecules involved in Akt1-regulated cell proliferation.

In addition to the effect of Akt1 on tumor cell growth, its effects on tumor cell migration and invasion are of considerable importance. In the present study, we demonstrated that upregulation of Akt1 expression in liver cancer HepG2 cells markedly promoted the wound closure and the number of migrated cells. Moreover, enforced expression of Akt1 also significantly enhanced the invasion of HepG2 cells. Treatment with wortmannin significantly attenuated the positive effect of Akt1 on HepG2 cell migration and invasion. Then, we examined whether Akt1 plays a similar role in HCT 116 cells. Notably, the results from the wound healing assay suggested that overexpression of Akt1 significantly inhibited the wound closure of HCT 116 cells, while wortmannin partially rescued the inhibitory effect of Akt1 on colorectal tumor HCT 116 cells. In addition, the results from the Transwell migration assay also verified that enforced Akt1 expression significantly reduced HCT 116 cell migration compared with the corresponding control group. Furthermore, we also discovered that upregulation of Akt1 expression significantly inhibited HCT 116 cell invasion. However, following treatment with wortmannin, the inhibitory effect of Akt1 on HCT 116 cell migration and invasion was considerably alleviated. Considering the completely inverse effect of Akt1 on the migration and invasion in HepG2 and HCT 116 cells, we propose that the potential molecular mechanisms may be different in these two types of cell lines.

Matrix metalloproteinases (MMPs) are a large family of enzymes, which play a fundamental role in various components of the extracellular matrix degradation and remodeling (32). 
MMPs have been found to be overexpressed in several human tumors and correlate with advanced stage, invasion, metastatic properties and poor prognosis (33). Among secreted MMPs, MMP2 and MMP9 are well known to play a pivotal role in tumor invasion and metastasis development in several types of cancer including hepatocellular and colorectal carcinomas $(22,23)$. Moreover, it has been shown that silencing of Akt1 is associated with reduced expression of MMP2 and MMP9 in both SGC7901 gastric adenocarcinoma and U251 glioma cells (34). Consistent with these reports, our results also showed that upregulation of Akt1 resulted in increased expression of MMP2 and MMP9 in HepG2 cells, while it inhibited the expression of MMP2 and MMP9 in colorectal carcinoma HCT 116 cells. Treatment with pharmacologic inhibitor of PI3K completely reversed the effect of Akt1 in both cell types. In addition, the protein levels of HIF1 $\alpha$ and VEGF were also significantly increased in HepG2-Akt1 cells compared to its control; by contrast, they were degraded in HCT 116 cells. Upon wortmannin treatment, the expression of HIF1 $\alpha$ and VEGF was strongly suppressed in HepG2 cells, whereas it was upregulated in HCT 116 cells. Consistent with these results, activation of the PI3K/Akt1 pathway in tumor cells resulted in increased VEGF secretion, both by HIF-1 $\alpha$-dependent and -independent mechanisms. Moreover, sustained endothelial activation of Akt1 has been shown to induce the formation of structurally abnormal blood vessels (35). Furthermore, silencing of Akt1 by siRNA markedly decreased HIF-1 $\alpha$ translation in normoxia in the presence of dimethyloxallyl glycine and in hypoxia (36). Collectively, these findings suggested that several signaling molecules, including MMP2, MMP9, HIF1 $\alpha$ and VEGF, may play a key role in Akt1-mediated cell migration and invasion. Meanwhile, we proposed that Akt1 upregulation may first promote the expression of MMP2, MMP9, HIF1 $\alpha$ and VEGF, which contributed to inducing the migration and invasion of HepG2 cells. In contrast, in colorectal carcinoma HCT 116 cells, upregulation of Akt1 suppressed the expression of these genes, which resulted in reduced cell migration and invasion. However, how Akt1 regulates these multiple molecules remains to be further investigated.

In the present study, we demonstrated that upregulation of Akt1 promotes cell proliferation in both HepG2 and HCT 116 cells, while the role of Akt1 in cell migration and invasion is completely distinct in these two cell types, suggesting the role of Akt1 in cell proliferation is independent of cell migration and invasion, although all are malignant phenotypes in the process of tumor development. Consistent with our observations, Pierau et al (7) demonstrated that constitutive Akt1 signals attenuate $\mathrm{B}$-cell receptor signaling and proliferation, but enhance B-cell migration and effector function. Moreover, our results also demonstrated that overexpression of Aktl promoted the cell migration and invasion of human liver cancer HepG2 cells; however, it attenuated the cell migration and invasion of human colorectal carcinoma HCT 116 cells. Therefore, we proposed that the effect of Akt1 on cell migration and invasion is likely due to the difference in cell type and context. Indeed, it has been shown that human non-small cell lung cancer (NSCLC) A549 cells with an expression construct for Akt1, exhibited significantly higher invasive ability through Matrigel than those cells with a control empty vector (37). Knockdown of Akt1 expression in NSCLC cells resulted in decreased cell migration (38). Furthermore, high levels of total Akt1 were also associated with increased lymph node metastasis in human prostate cancer (39). In the present study, ectopic expression of Akt1 significantly promoted the cell proliferation, migration and invasion in human liver cancer HepG2 cells, suggesting that Akt1 is may be a promising target for human liver cancer therapy.

On the other hand, Yoeli-Lerner et al (12) revealed that expression of activated Akt1 potently blocked the cell migration and invasion through Matrigel in three distinct breast cancer cell lines. Irie et al (13) also discovered that silencing of Akt1 expression markedly enhanced cell migration induced by growth factor in MCF-10A cells. Consistent with these observations, in the present study, upregulation of Aktl expression significantly inhibited the cell migration and invasion in colorectal carcinoma HCT 116 cells. Furthermore, in vivo evidence also identified the role for Akt1 in blunting breast cancer cell invasion and subsequent metastasis. Using a mouse bitransgenic assay of mammary tumorigenesis with constitutively activated Akt1 and ErbB-2, Hutchinson et al (15) reported that upregulation of activated Akt1 expression resulted in a decrease in the incidence of metastatic lesions compared with control animals.

Although we, and other groups, have demonstrated the inhibitory effect of Akt1 on cell migration and invasion in several types of tumor cells, the precise cell signaling mechanisms remain to be further investigated. Multiple molecular mechanisms have been shown to be involved in Akt1-mediated negative regulation of cell migration and invasion, including inhibition of NFAT transcription factor (12) or phosphorylation of paladin (11), activation of ERK signaling pathway (13) and cell surface B1-integrins (14). Of note, silencing of AKT1 in PC-3 cells also resulted in strong upregulation of VEGFR2 (14). Consistently, in the present study, we directly demonstrated that enforced expression of Akt1 significantly not only reduced the expression of VEGF, but also suppressed the expression of MMP2, MMP9 and HIF1 $\alpha$, which then suppressed the migration and invasion of HCT 116 cells.

In the present study, our experiments on Akt1 were limited to human hepatocellular carcinoma HepG 2 cells and colorectal carcinoma HCT 116 cells. Enforced Akt1 expression significantly increased cell proliferation through induction of $\mathrm{Bcl}-2$ and NF- $\kappa$ B in both HepG2 and HCT 116 cells. Moreover, our observations also showed that Akt1 overexpression significantly promoted the expression of MMP2, MMP9, HIF1 $\alpha$ and VEGF, which then contributed to enhancing the migration and invasion of HepG2 cells. Upregulation of Akt1 expression markedly downregulated the levels of these proteins and suppressed the migration and invasion of HCT 116 cells. In order to verify these effects of Akt1, more types of liver cancer and colorectal carcinoma cell lines are required. Furthermore, the precise molecular mechanisms remain to be further investigated.

Although Akt1 contributes to proliferation in both types of tumor cells, specific pharmacological inhibition may have a differential impact on migration and invasion in different cell types. Understanding these differences is crucial to the implication of specific inhibitors for cancer therapies. 


\section{Acknowledgements}

The present study was supported by the NSFC-Henan 'Talented Man' Train Union Fund (no. U1204829) and the Projects of Science and Technology of Henan (no. 102300410095).

\section{References}

1. Staal SP: Molecular cloning of the akt oncogene and its human homologues AKT1 and AKT2: amplification of AKT1 in a primary human gastric adenocarcinoma. Proc Natl Acad Sci USA 84: 5034-5037, 1987.

2. Jiang BH, Aoki M, Zheng JZ, Li J and Vogt PK: Myogenic signaling of phosphatidylinositol 3-kinase requires the serinethreonine kinase Akt/protein kinase B. Proc Natl Acad Sci USA 96: 2077-2081, 1999.

3. Fernandez-Hernando C, Jozsef L, Jenkins D, Di Lorenzo A and Sessa WC: Absence of Akt1 reduces vascular smooth muscle cell migration and survival and induces features of plaque vulnerability and cardiac dysfunction during atherosclerosis. Arterioscler Thromb Vasc Biol 29: 2033-2040, 2009.

4. Menges CW, Sementino E, Talarchek J, et al: Group I p21-activated kinases (PAKs) promote tumor cell proliferation and survival through the AKT1 and Raf-MAPK pathways. Mol Cancer Res 10: 1178-1188, 2012.

5. Okumura N, Yoshida H, Nishimura Y, Kitagishi Y and Matsuda S Terpinolene, a component of herbal sage, downregulates AKT1 expression in K562 cells. Oncol Lett 3: 321-324, 2012.

6. Young CD, Nolte EC, Lewis A, Serkova NJ and Anderson SM Activated Akt1 accelerates MMTV-c-ErbB2 mammary tumourigenesis in mice without activation of ErbB3. Breast Cancer Res 10: R70, 2008.

7. Pierau M, Na SY, Simma N, et al: Constitutive Akt1 signals attenuate B-cell receptor signaling and proliferation, but enhance B-cell migration and effector function. Eur J Immunol 42: 3381-3393, 2012

8. Siegel R, Ward E, Brawley O and Jemal A: Cancer statistics, 2011: the impact of eliminating socioeconomic and racial disparities on premature cancer deaths. CA Cancer J Clin 61: 212-236, 2011.

9. Sporn MB: The war on cancer. Lancet 347: 1377-1381, 1996.

10. Kim EK, Yun SJ, Ha JM, et al: Selective activation of Akt1 by mammalian target of rapamycin complex 2 regulates cancer cel migration, invasion, and metastasis. Oncogene 30: 2954-2963, 2011.

11. Chin YR and Toker A: The actin-bundling protein palladin is an Akt1-specific substrate that regulates breast cancer cell migration. Mol Cell 38: 333-344, 2010.

12. Yoeli-Lerner M, Yiu GK, Rabinovitz I, Erhardt P, Jauliac S and Toker A: Akt blocks breast cancer cell motility and invasion through the transcription factor NFAT. Mol Cell 20: 539-550, 2005.

13. Irie HY, Pearline RV, Grueneberg D, et al: Distinct roles of Akt1 and Akt2 in regulating cell migration and epithelialmesenchymal transition. J Cell Biol 171: 1023-1034, 2005.

14. Virtakoivu R, Pellinen T, Rantala JK, Perala M and Ivaska J: Distinct roles of AKT isoforms in regulating $\beta 1$-integrin activity, migration, and invasion in prostate cancer. Mol Biol Cell 23: $3357-3369,2012$

15. Hutchinson JN, Jin J, Cardiff RD, Woodgett JR and Muller WJ: Activation of Akt-1 (PKB- $\alpha$ ) can accelerate ErbB-2-mediated mammary tumorigenesis but suppresses tumor invasion. Cancer Res 64: 3171-3178, 2004.

16. Maroulakou IG, Oemler W, Naber SP and Tsichlis PN: Akt1 ablation inhibits, whereas Akt2 ablation accelerates, the development of mammary adenocarcinomas in mouse mammary tumor virus (MMTV)-ErbB2/neu and MMTV-polyoma middle T transgenic mice. Cancer Res 67: 167-177, 2007.

17. Xie SQ, Li Q, Zhang YH, et al: NPC-16, a novel naphthalimidepolyamine conjugate, induced apoptosis and autophagy in human hepatoma HepG2 cells and Bel-7402 cells. Apoptosis 16: 27-34, 2011.

18. Xu T, Zhu Y, Xiong Y, Ge YY, Yun JP and Zhuang SM: MicroRNA-195 suppresses tumorigenicity and regulates G1/S transition of human hepatocellular carcinoma cells. Hepatology 50: 113-121, 2009.
19. Koomoa DL, Geerts D, Lange I, et al: DFMO/eflornithine inhibits migration and invasion downstream of MYCN and involves p2 $7^{\text {Kipl }}$ activity in neuroblastoma. Int J Oncol 42: 1219-1228, 2013.

20. Fang JH, Zhou HC, Zeng C, et al: MicroRNA-29b suppresses tumor angiogenesis, invasion, and metastasis by regulating matrix metalloproteinase 2 expression. Hepatology 54: 1729-1740, 2011

21. Xie SQ, Zhang YH, Li Q, et al: 3-Nitro-naphthalimide and nitrogen mustard conjugate NNM-25 induces hepatocellular carcinoma apoptosis via PARP-1/p53 pathway. Apoptosis 17: 725-734, 2012.

22. Li J, Lau GK, Chen L, et al: Interleukin 17A promotes hepatocellular carcinoma metastasis via NF- $\mathrm{BB}$ induced matrix metalloproteinases 2 and 9 expression. PLoS One 6: e21816, 2011.

23. Yang P, Yuan W, He J, et al: Overexpression of EphA2, MMP-9, and MVD-CD34 in hepatocellular carcinoma: implications for tumor progression and prognosis. Hepatol Res 39: 1169-1177, 2009.

24. Chandarlapaty S, Sawai A, Scaltriti M, et al: AKT inhibition relieves feedback suppression of receptor tyrosine kinase expression and activity. Cancer Cell 19: 58-71, 2011.

25. Dillon RL and Muller WJ: Distinct biological roles for the akt family in mammary tumor progression. Cancer Res 70: 4260-4264, 2010.

26. Cariaga-Martinez AE, Lopez-Ruiz P, Nombela-Blanco MP, et al: Distinct and specific roles of AKT1 and AKT2 in androgensensitive and androgen-independent prostate cancer cells. Cell Signal 25: 1586-1597, 2013.

27. Saji M, Narahara K, McCarty SK, et al: Akt1 deficiency delays tumor progression, vascular invasion, and distant metastasis in a murine model of thyroid cancer. Oncogene 30: 4307-4315, 2011.

28. Imanishi Y, Hu B, Xiao G, Yao X and Cheng SY: Angiopoietin-2, an angiogenic regulator, promotes initial growth and survival of breast cancer metastases to the lung through the integrin-linked kinase (ILK)-AKT-B cell lymphoma 2 (Bcl-2) pathway. J Biol Chem 286: 29249-29260, 2011.

29. Yang L, Xiao L, Ma X, et al: Effect of DNAzymes targeting Akt1 on cell proliferation and apoptosis in nasopharyngeal carcinoma. Cancer Biol Ther 8: 366-371, 2009.

30. Basseres DS and Baldwin AS: Nuclear factor- $\kappa$ B and inhibitor of $\kappa \mathrm{B}$ kinase pathways in oncogenic initiation and progression. Oncogene 25: 6817-6830, 2006.

31. Han SS, Yun H, Son DJ, et al: NF- $\mathrm{KB} / \mathrm{STAT} 3 / \mathrm{PI} 3 \mathrm{~K}$ signaling crosstalk in iMyc ${ }^{\mathrm{E} \mu} \mathrm{B}$ lymphoma. Mol Cancer 9: 97, 2010.

32. Egeblad $M$ and Werb Z: New functions for the matrix metalloproteinases in cancer progression. Nat Rev Cancer 2: 161-174, 2002.

33. Rundhaug JE: Matrix metalloproteinases and angiogenesis. J Cell Mol Med 9: 267-285, 2005.

34. Zhang J,Zhang QY, Fu YC, et al: Expression of p-Akt and COX-2 in gastric adenocarcinomas and adenovirus mediated Akt1 and COX-2 ShRNA suppresses SGC-7901 gastric adenocarcinoma and U251 glioma cell growth in vitro and in vivo. Technol Cancer Res Treat 8: 467-478, 2009.

35. Karar J and Maity A: PI3K/AKT/mTOR pathway in angiogenesis. Front Mol Neurosci 4: 51, 2011.

36. Pore N, Jiang Z, Shu HK, Bernhard E, Kao GD and Maity A: Akt1 activation can augment hypoxia-inducible factor-1 $\alpha$ expression by increasing protein translation through a mammalian target of rapamycin-independent pathway. Mol Cancer Res 4: 471-479, 2006.

37. Lee YC, Lin HH, Hsu CH, Wang CJ, Chiang TA and Chen JH: Inhibitory effects of andrographolide on migration and invasion in human non-small cell lung cancer A549 cells via downregulation of PI3K/Akt signaling pathway. Eur J Pharmacol 632: 23-32, 2010.

38. Lee MW, Kim DS, Lee JH, et al: Roles of AKT1 and AKT2 in non-small cell lung cancer cell survival, growth, and migration. Cancer Sci 102: 1822-1828, 2011

39. Li R, Dai H, Wheeler TM, et al: Prognostic value of Akt-1 in human prostate cancer: a computerized quantitative assessment with quantum dot technology. Clin Cancer Res 15: 3568-3573, 2009. 